\title{
Nerve growth factor supplementation reverses the impairment, induced by Type 1 diabetes, of hindlimb post-ischaemic recovery in mice
}

\author{
M. B. Salis ${ }^{1}$ G. Graiani ${ }^{1,3}$ - E. Desortes ${ }^{1}$ R. B. Caldwell ${ }^{4}$ P. Madeddu $^{1,5}$ - C. Emanueli ${ }^{1,2}$ \\ ${ }^{1}$ Experimental Medicine and Gene Therapy Unit, National Institute of Biostructures and Biosystems, Osilo and Alghero, Italy \\ 2 Molecular and Cellular Medicine Laboratory, National Institute of Biostructures and Biosystems \\ (I.N.B.B. Inter-University Consortium), Porto Conte Ricerche, Alghero (Sassari), Italy \\ ${ }^{3}$ Department of Pathology, University of Parma, Parma, Italy \\ ${ }^{4}$ Vascular Biology Centre, Medical College of Georgia, Augusta, Georgia, USA \\ ${ }^{5}$ Department of Internal Medicine, University of Sassari, Sassari, Italy
}

\section{Abstract}

Aims/hypothesis. Type 1 diabetes increases the risk of peripheral ischaemia and impairs recovery once ischaemia occurs, probably because the healing process is hampered by diabetes-induced endothelial dysfunction. In normoglycaemic mice subjected to limb ischaemia, blockade of nerve growth factor (NGF) compromises reparative angiogenesis. In the present study, we evaluated if expressional alterations of endogenous NGF system components are associated with diabetesrelated impairment in neovascularisation. In addition, we tested whether the correction of NGF liabilities benefits post-ischaemic healing of Type 1 diabetic animals.

Methods. Unilateral hindlimb ischaemia was produced in streptozotocin-induced Type 1 diabetic mice. Purified murine NGF (20 $\mu \mathrm{g}$ daily for 14 days) or PBS were injected into ischaemic adductors. Non-diabetic mice given PBS served as controls. Hindlimb blood flow was analysed sequentially for up to 14 days. At necroscopy, adductors were removed for quantification of microvessel density, endothelial cell apoptosis and NGF receptor expression. NGF content was deter- mined by ELISA three days after ischaemia. In vitro, we tested whether NGF protects endothelial cells from apoptosis induced by high glucose and whether vascular endothelial growth factor-A (VEGF-A) is involved in this beneficial effect.

Results. Muscles removed from Type 1 diabetic mice showed reduced NGF content and up-regulation of the NGF p75 receptor. NGF supplementation promoted capillarisation and arteriogenesis, reduced apoptosis, and accelerated blood flow recovery. NGF stimulated VEGF-A production by human endothelial cells incubated in high-glucose medium and conferred resistance against high-glucose-induced apoptosis via a VEGF-A-mediated mechanism.

Conclusions/interpretation. NGF protects endothelial cells from apoptosis induced by Type 1 diabetes and facilitates reparative neovascularisation. The findings may open up new therapeutic options for the treatment of diabetic complications.

Keywords Apoptosis - Endothelial cell - Ischaemia · Neovascularisation $\cdot \mathrm{NGF} \cdot$ Skeletal muscle $\cdot$ Type 1 diabetes mellitus
Received: 31 December 2003 / Accepted: 7 March 2004

Published online: 8 June 2004

(C) Springer-Verlag 2004

C. Emanueli (৫)

Molecular and Cellular Medicine Laboratory,

National Institute of Biostructures and Biosystems

(I.N.B.B. Inter-University Consortium), Porto Conte Ricerche,

SP55 Porto Conte-Capo Caccia-Località Tramariglio,

07040 Alghero (Sassari), Italy

E-mail: emanueli@yahoo.com

Tel.: +39-79-3441006, Fax: +39-79-3441006

\section{Introduction}

Peripheral ischaemia is more common and severe among diabetic patients than among people who do not have diabetes $[1,2]$. The clinical outcome, more-

Abbreviations: DAPI, 4'-6'-diaminidino phenylindole $\cdot$ NGF, nerve growth factor $\cdot \mathrm{p} 75, \mathrm{P} 75$ receptor of NGF $\cdot \mathrm{SCP} / \mathrm{DAB}$, streptavidin-conjugated peroxidase $\cdot$ STZ, streptozotocin . TrkA, tyrosine kinase receptor-A of NGF - TUNEL, terminal deoxynucleotidyl transferase-mediated dUTP nick-end labelling - VEGF-A, vascular endothelial growth factor-A 
over, following vascular occlusion is uniquely poor in diabetic patients, as collateralisation is inadequate. An insufficient surge of vascular growth factors might contribute to the deficit [3].

The neurotrophin nerve growth factor (NGF), initially recognised to be involved in nerve regeneration, also elicits a pro-angiogenic activity and promotes endothelial cell survival [4] and proliferation [5]. In skeletal muscles of non-diabetic mice, local expression of endogenous NGF and of its pro-angiogenic receptor tyrosine kinase receptor-A (TrkA) increases after blood flow is interrupted, and blockade of this native reaction results in severely compromised capillary growth in response to ischaemia [4]. Conversely, NGF supplementation accelerates tissue perfusion recovery by potentiating angiogenesis and arteriogenesis [4].

We therefore hypothesised that this supply-side approach could have special therapeutic value in Type 1 diabetes mellitus. In fact, extensive previous studies have demonstrated that diabetes reduces the levels of NGF in several peripheral tissues, including nerves $[6,7,8]$ and limb skeletal muscles [9].

The objective of the present study was two-fold: (i) to investigate whether ischaemic limb muscles of Type 1 diabetic mice lack the ability to produce adequate amounts of NGF, and (ii) to investigate the therapeutic potential of exogenous NGF supplements for the treatment of ischaemic complications related to Type 1 diabetes.

\section{Materials and methods}

Induction and assessment of Type 1 diabetes. Procedures complied with the standards stated in the Guide for the Care and Use of Laboratory Animals (Institute of Laboratory Animal Resources, National Academy of Sciences, Bethesda, Md., USA 1996) and were in agreement with Italian law.

Type 1 diabetes was induced by streptozotocin (STZ) (40 $\mathrm{mg} / \mathrm{kg}$ body weight i.p. daily for 5 consecutive days) in 2-month-old male CD1 mice (Charles River, Calco, Italy). In the majority of treated mice (around 85\%) this procedure creates a moderate diabetic condition that allows them to survive for up to 4 months after treatment without receiving insulin supplementation [10] and without experiencing significant impairment of body weight gain in the first 2 months after STZ treatment (Emanueli et al., unpublished data). Mice that had fasting glycaemia higher than $13.77 \mathrm{mmol} / \mathrm{l}$ and glycosuria at 14 days after the first STZ injection were considered diabetic. After this, urinary glucose levels were checked every 14 days, and only mice showing overt glycosuria on all occasions were included in the study. Body weight was recorded before STZ treatment and every 7 days thereafter.

CD1 mice that were matched for age and sex and had not received STZ were used as non-diabetic controls. The body weight of non-diabetic control animals was recorded during the experimental period in the same way as in the Type 1 diabetic mice.

Induction of hindlimb ischaemia and nerve growth factor treatment. At 1 month from diabetes onset, mice were submitted to unilateral hindlimb ischaemia by electro-coagulation of the up- per part of the left femoral artery. This was done under anaesthesia (2,2,2-tribromoethanol, $880 \mathrm{mmol} / \mathrm{kg}$ i.p., Sigma, Milan, Italy). Diabetic mice were randomly allocated to receive NGF $(20 \mu \mathrm{g} / 20 \mu \mathrm{l}$ per day into the ischaemic adductor muscle, $n=10$ mice) or its vehicle PBS $(n=12)$. Treatments started on the day of surgery and were continued for the entire duration of the experiment (14 days). Non-diabetic control mice $(n=8)$ underwent the same surgical procedure and received intramuscular PBS.

Nerve growth factor system expression in diabetic ischaemic muscles. A commercial ELISA kit (Promega, Milan, Italy) was used to determine NGF content in homogenates of ischaemic and contralateral adductor muscles removed 3 days after induction of ischaemia in Type 1 diabetic mice $(n=8)$ or nondiabetic controls $(n=7)$.

The expression of TrkA and P75 receptor of NGF (p75) in ischaemic and contralateral adductors was evaluated by immunohistochemistry at 14 days after surgery in Type 1 diabetic $(n=4)$ and non-diabetic $(n=5)$ mice. In addition, the effect of NGF supplementation or PBS on TrkA and p75 expression was determined in diabetic muscles ( $n=6$ per group). To do this, anaesthetised mice were first perfused with heparinased PBS (1 min) and then with $10 \%$ buffered formalin $(10 \mathrm{~min})$ at $100 \mathrm{~mm} \mathrm{Hg}$ via a cannula inserted into the abdominal aorta. Ischaemic and contralateral muscles were then removed and processed for paraffin embedding. TrkA identification was performed on 3- $\mu$ m-thick muscular sections by using a polyclonal rabbit antibody (1:50, Santa Cruz, Santa Cruz, Calif., USA) diluted in PBS containing 1\% normal goat serum. The specific binding was detected using a biotinylated anti-rabbit IgG and the streptavidin-peroxidase technique (Vectastain Elite ABC kit, Vector Laboratories, Burlingame, Calif., USA). P75 receptor was identified by using a monoclonal mouse antibody (1:50, DAKO, Carpinteria, Calif., USA) diluted in PBS containing $1 \%$ normal goat serum. Sections were incubated at the same time with primary and secondary antibody (DAKO), stained with streptavidin-conjugated peroxidase (SCP/DAB) and counterstained with haematoxylin. The primary antibody was not included in negative controls. Sections were evaluated at $1000 \times$. In each field, the number of TrkA-positive endothelial cells, TrkA-positive myocytes, p75-positive endothelial cells and p75-positive myocytes was separately counted to compute their density per $\mathrm{mm}^{2}$ of section. The number of TrkA-positive endothelial cells or p75-positive endothelial cells per 1000 capillaries was also calculated.

Effect of nerve growth factor supplementation on neovascularisation and apoptosis. We evaluated the microvascular density at 14 days after surgery in ischaemic and contralateral adductors of Type 1 diabetic mice receiving NGF or PBS. The same analysis was performed in muscles removed from PBS-treated non-diabetic controls. We cut 5 - $\mu$ m-thick sections from each sample with muscle fibres transversely oriented, and stained them with haematoxylin and eosin. Capillary and myofibre densities per $\mathrm{mm}^{2}$ of muscular section were determined as previously described [10]. In addition, capillary density was normalised to myofibre density $\left(\mathrm{n}_{\text {cap }} / \mathrm{n}_{\text {fiber }}\right)$ [10]. Arteriole density per $\mathrm{mm}^{2}$ of section area $\left(\mathrm{n}_{\text {art }} / \mathrm{mm}^{2}\right)$ was also calculated as described [10].

Apoptosis was assessed by terminal deoxynucleotidyl transferase-mediated dUTP nick-end labelling (TUNEL) assay in left and right adductors of NGF or PBS-treated Type 1 diabetic mice ( $n=5$ for each group). Sections 3 - $\mu$ m-thick were stained with SCP/DAB and counterstained with haematoxylin. Negative controls were incubated in the absence of the terminal deoxynucleotidyl transferase. Sections were examined at 
1000× magnification. TUNEL-positive endothelial cells and muscular cells were separately counted. The apoptotic rate was expressed as the number of TUNEL-positive cells per $\mathrm{mm}^{2}$ of tissue. Apoptosis of endothelial cells was also computed as the number of apoptotic endothelial cells per 1000 capillaries.

Hindlimb blood flow measurements. Foot blood flow was measured at $30 \mathrm{~min}, 7$ days, and 14 days after ischaemia using a perfusion imager system (Lisca, Linkoping, Sweden). The ischaemic : non-ischaemic foot blood flow ratio was calculated as an index of perfusion recovery [4].

Evaluation of retinal vasculature of Type 1 diabetic mice receiving intramuscular NGF. In order to evaluate the possible occurrence of side effects similar to retinopathy in connection with NGF supplementation, we studied the retinal vasculature of Type 1 diabetic mice whose ischaemic limb muscles had been injected with NGF or PBS for 14 days ( $n=5$ per group). Anaesthetised mice were perfusion-fixed through the heart and their eyes were removed, washed in PBS and fixed in $4 \%$ formalin. Mouse retinas were mounted flat on glass slides and the retinal vasculature was labelled with biotinylated Griffonia simplicifolia lectin B4 (Vector Laboratories), followed by Texas Red-conjugated Avidin D (Vector Laboratories) [11, 12]. The specimens were coded and four microscopic images were collected from both the central and peripheral region of each retina. Images were evaluated for signs of vascular pathology by two investigators blinded to the experimental treatment conditions.

Anti-apoptotic effect of nerve growth factor on cultured endothelial cells. HUVECs were cultured in M199 with 20\% FBS, $0.8 \%$ bovine brain extract (Clonetics, Walkersville, Md., USA) and antibiotics.

The effect of NGF on apoptosis induced by a combination of serum deprivation and high glucose was evaluated. To this aim, HUVECs prepared at our laboratory were re-suspended in full normoglycidic medium and seeded $\left(10^{4}\right.$ cells per well $)$ on to 12 -well plates. After $24 \mathrm{~h}$, the medium was changed into M199 containing $30 \mathrm{mmol} / \mathrm{l} \mathrm{D}$-glucose and $0.1 \% \mathrm{FBS}$. After an additional $2 \mathrm{~h}$, NGF (100 ng/ml) or PBS was added and incubated for $24 \mathrm{~h}$. The same experiment was repeated in the presence of an antibody $(500 \mathrm{ng} / \mathrm{ml})$ that neutralises vascular endothelial growth factor-A (VEGF-A) or control IgG.

Conditioned media were removed and stored at $-20{ }^{\circ} \mathrm{C}$ for subsequent assay, while attached cells were washed and fixed with $4 \%$ paraformaldehyde. Cells were labelled with $4^{\prime}-6^{\prime}$ diaminidino phenylindole (DAPI) (100 ng/100 $\mu \mathrm{l}$, Sigma), and apoptotic cells were identified on the basis of the appearance of nuclei with condensed and fragmented chromatin. DAPIstained cells were examined by fluorescent microscopy at $20 \times$. In each well, five randomly selected fields were captured with a videocamera (Zeiss, Milan, Italy) and automatically analysed by Image Proplus software (Carlsbad, Calif., USA) for total cell number and apoptotic cell number. For each field, the percentage of apoptotic cells was calculated. This experiment was performed in quadruplicate and repeated three times.

Assay of vascular endothelial growth factor-A content in conditioned media of NGF-treated HUVECs. The content of human VEGF-A in the conditioned media collected during the experiment described above was measured by a dedicated ELISA kit (human VEGF quantikine, R\&D Systems, Minneapolis, Minn., USA).

Statistical analysis. Data are expressed as means \pm SEM. Multivariate repeated-measures ANOVA was performed to test for interaction between treatments and groups. In multiple comparisons among independent groups in which ANOVA and the $F$ test indicated significant differences the statistical value was determined according to Bonferroni's method. Differences within and between groups were determined using paired or unpaired Student's $t$ tests respectively. A $p$ value of less than 0.05 was considered to denote statistical significance.

\section{Results}

Expression of nerve growth factor receptor. No significant change in body weight was observed within each group during the study. Furthermore, at death, the body weight of the mice was similar in all groups (non-diabetic: $33.0 \pm 0.4 \mathrm{~g}$; PBS-treated diabetic: $32.2 \pm 1.1 \mathrm{~g}$; NGF-treated diabetic: $32.5 \pm 1.6 \mathrm{~g} ; p=\mathrm{NS}$ for all comparisons).

As shown in Figure 1, diabetes reduced NGF content in ischaemic and normally perfused muscles.

We also found (Fig. 2) that the number of p75-positive endothelial cells was greater in ischaemic diabetic muscles than in non-diabetic ones $(43.4 \pm 12.4$ vs $1.5 \pm 0.5$ positive endothelial cells per 1000 capillaries respectively, $p<0.01)$. P75-positive myocytes were also augmented in diabetic muscles $(5.5 \pm 3.3$ positive myocytes per $\mathrm{mm}^{2}$ vs $0.9 \pm 0.3$ in non-diabetic controls, $p<0.05)$. Diabetes did not alter TrkA receptor expression at the level of endothelial cells $(17.8 \pm 5.2$ TrkA-positive endothelial cells per 1000 capillaries vs

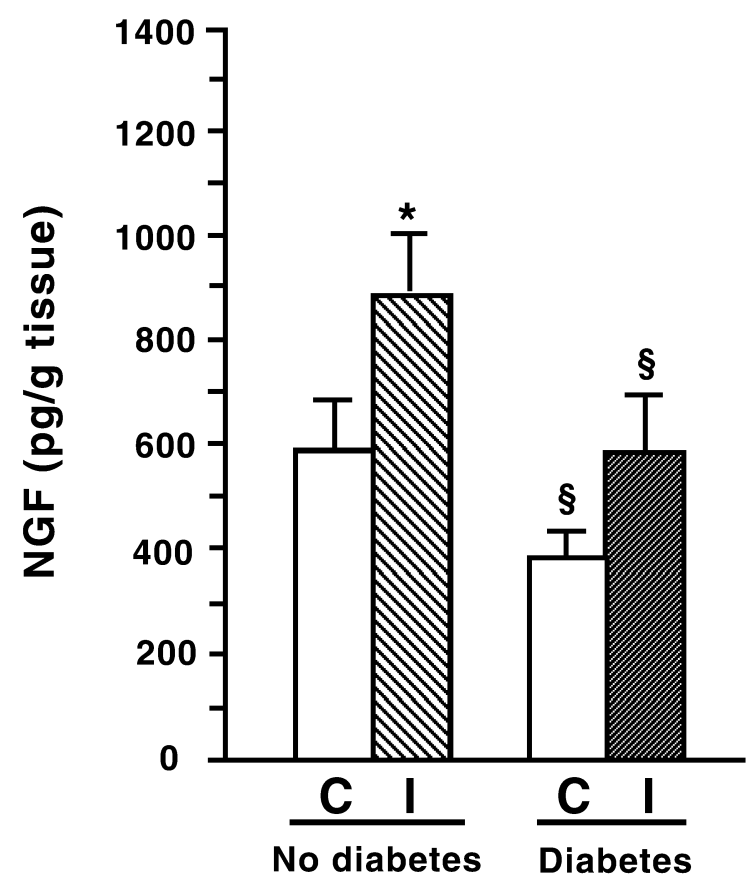

Fig. 1. Immunoreactive nerve growth factor (NGF) content in ischaemic (I) and contralateral (C) adductor muscles removed from Type 1 diabetic mice or normoglycaemic controls (No diabetes) 3 days after induction of unilateral ischaemia. Values are means \pm SEM. ${ }^{*} p<0.05$ vs contralateral; ${ }^{\S} p<0.05$ vs No diabetes 
a
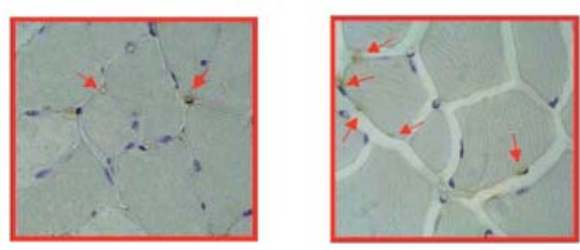

b

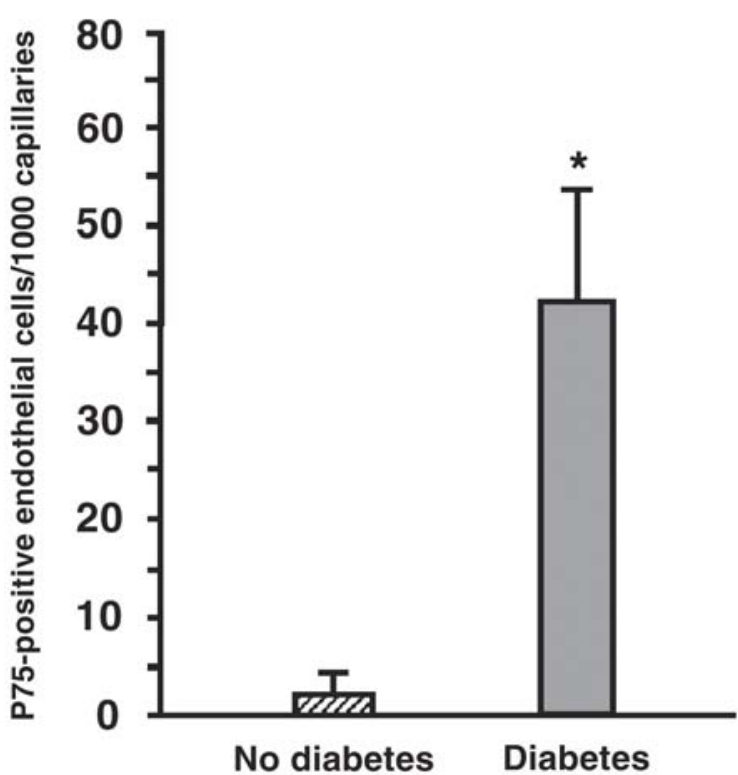

Fig. 2. The images (a) are representative of p75-staining in sections derived from ischaemic muscle of Type 1 diabetic mice (right) or non-diabetic controls (No Diabetes, left). Arrows: endothelial cells positive for p75 receptors; bar length: $10 \mu \mathrm{m}$; original magnification: $1000 \times$. The bar graph (b) shows the number of p75-receptor-positive endothelial cells per 1000 capillaries in sections obtained from ischaemic muscles removed from mice as in (a). Values are means \pm SEM. $* p<0.01$ vs No diabetes

$12.2 \pm 4.8$ in non-diabetic mice, $p=\mathrm{NS}$ ) or myocytes $\left(19.7 \pm 3.8\right.$ TrkA-positive myocytes per $\mathrm{mm}^{2}$ vs $13.2 \pm 3.5$ in non-diabetic mice, $p=\mathrm{NS}$ ).

Nerve growth factor stimulates reparative angiogenesis. As expected, in PBS-injected ischaemic muscles of non-diabetic control mice, capillary and arteriole density was increased by 31 and $46 \%$ indicative of reparative neovascularisation $(p<0.01$ vs normally perfused contralateral muscles for both comparisons). By contrast, both capillary formation (Fig. 3a, b) and arteriogenesis (Fig. 4) were depressed in PBS-injected ischaemic diabetic muscles ( $p=\mathrm{NS}$ vs contralaterals for both comparisons). However, a proper healing response was restored by daily injections of NGF. In fact, as shown in Figures 3a and 3b, NGF increased the capillary density of ischaemic muscles by $67 \%$ $\left(1145 \pm 77\right.$ vs $684 \pm 76 \mathrm{cap} / \mathrm{mm}^{2}$ in PBS-treated mice, $p<0.001)$ and the capillary : myofibre ratio by $40 \%$ $(1.4 \pm 0.1$ vs $1.0 \pm 0.1$ cap : fib in PBS-treated mice,

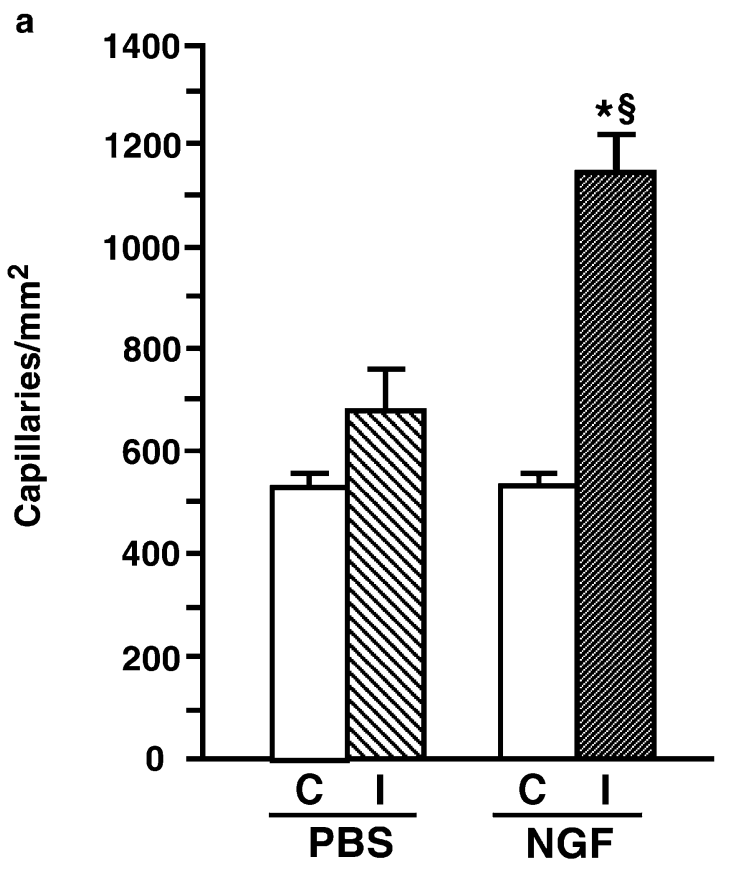

b

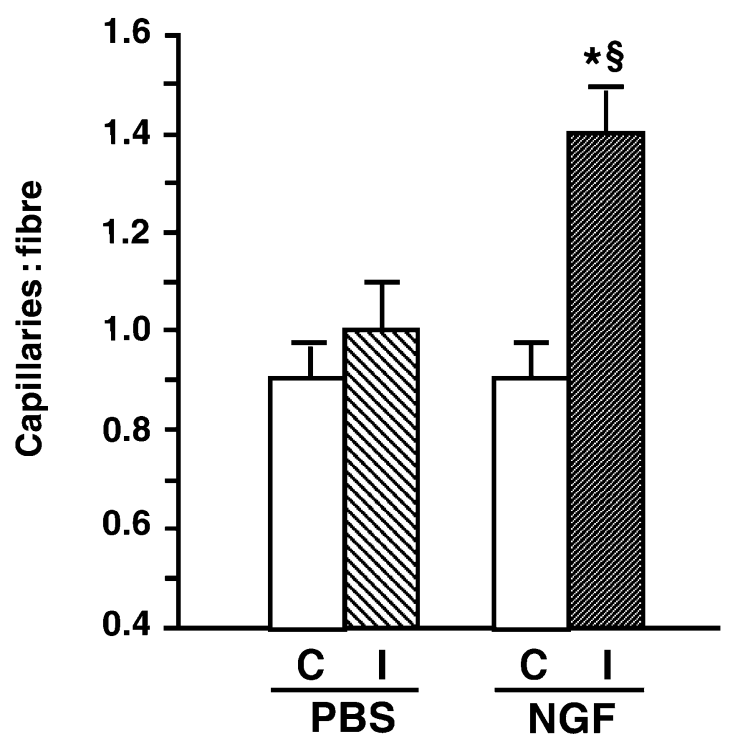

Fig. 3. The effect, in diabetic mice, of nerve growth factor (NGF) or PBS injection on capillary density of transverse muscular section (a) and capillary : myofibre ratio (b) in ischaemic adductor muscles (I). Non-ischaemic contralateral muscles (C) are shown for reference. Values are means \pm SEM. $* p<0.01$ vs contralaterals; ${ }^{\S} p<0.01$ vs PBS-treated ischaemic muscles

$p<0.01)$. Myofibre densitiy was not altered by NGF administration (data not shown). The therapeutic action of NGF was also evident (Fig. 4) at the level of the arterioles. In fact, NGF increased the density of arterioles in ischaemic muscles by $142 \%(13.6 \pm 2$ vs $5.6 \pm 0.4 \mathrm{art} / \mathrm{mm}^{2}$ in the PBS-treated group, $\left.p<0.01\right)$.

Nerve growth factor reduces apoptosis in diabetic ischaemic muscles. In diabetic ischaemic adductors, 


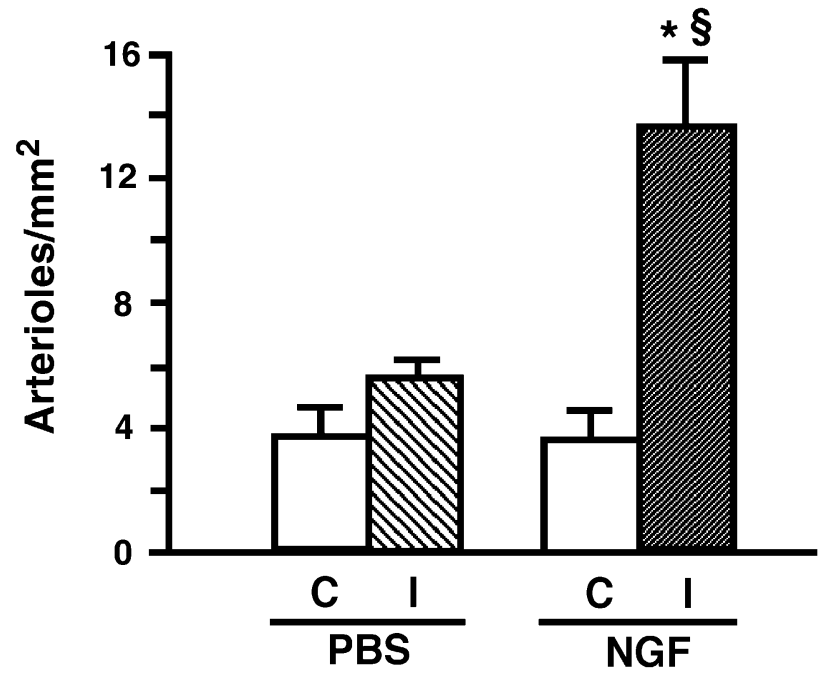

Fig. 4. The effect, in diabetic mice, of nerve growth factor (NGF) or PBS injection on arteriole density of ischaemic adductors (I). Values of contralateral muscles (C) are shown for reference. Values are means \pm SEM. $* p<0.01$ vs $C$; ${ }^{\S} p<0.01$ vs PBS-treated ischaemic muscles

endothelial cell and myofibre apoptosis was higher than in muscles of non-diabetic controls $(8.4 \pm 1.1$ vs $3.8 \pm 2.1$ apoptotic endothelial cells per $\mathrm{mm}^{2}$ and $15.3 \pm 3.3$ vs $2.8 \pm 3.9$ apoptotic myocytes per $\mathrm{mm}^{2}$, $p<0.05$ for both comparisons). In PBS-injected Type 1 diabetic muscles we observed (Fig. 5a, b) ischaemia-induced increases in apoptotic death both of endothelial cells $(p<0.01$ vs normally perfused contralateral muscles) and of myofibres $(p<0.05$ vs normally perfused contralateral muscles). Apoptosis was reduced by NGF supplementation to diabetic ischaemic muscles $(p<0.05$ vs PBS-injected diabetic ischaemic muscles; $p=\mathrm{NS}$ vs non-diabetic ischaemic muscles).

Nerve growth factor improves the post-ischaemic perfusion recovery of Type 1 diabetic mice. As shown in Figure 6, NGF reversed the diabetes-induced impairment of post-ischaemic blood flow recovery (left : right foot perfusion ratio at 14 days: $0.81 \pm 0.12$ vs $0.45 \pm 0.11$ in PBS-treated Type 1 diabetic mice, $p<0.05)$. The rate of perfusion recovery shown by NGF-treated Type 1 diabetic mice did not differ from that seen in non-diabetic control mice ( $p=\mathrm{NS}$ at all the time points).

Administration of nerve growth factor into the ischaemic adductor muscles does not affect retinal vasculature of Type 1 diabetic mice. As shown by representative images in Figure 7, the retinal vasculature of mice treated with NGF was morphologically normal and indistinguishable from that in the control mice. There was no evidence of microaneurysms or neovascular growth.
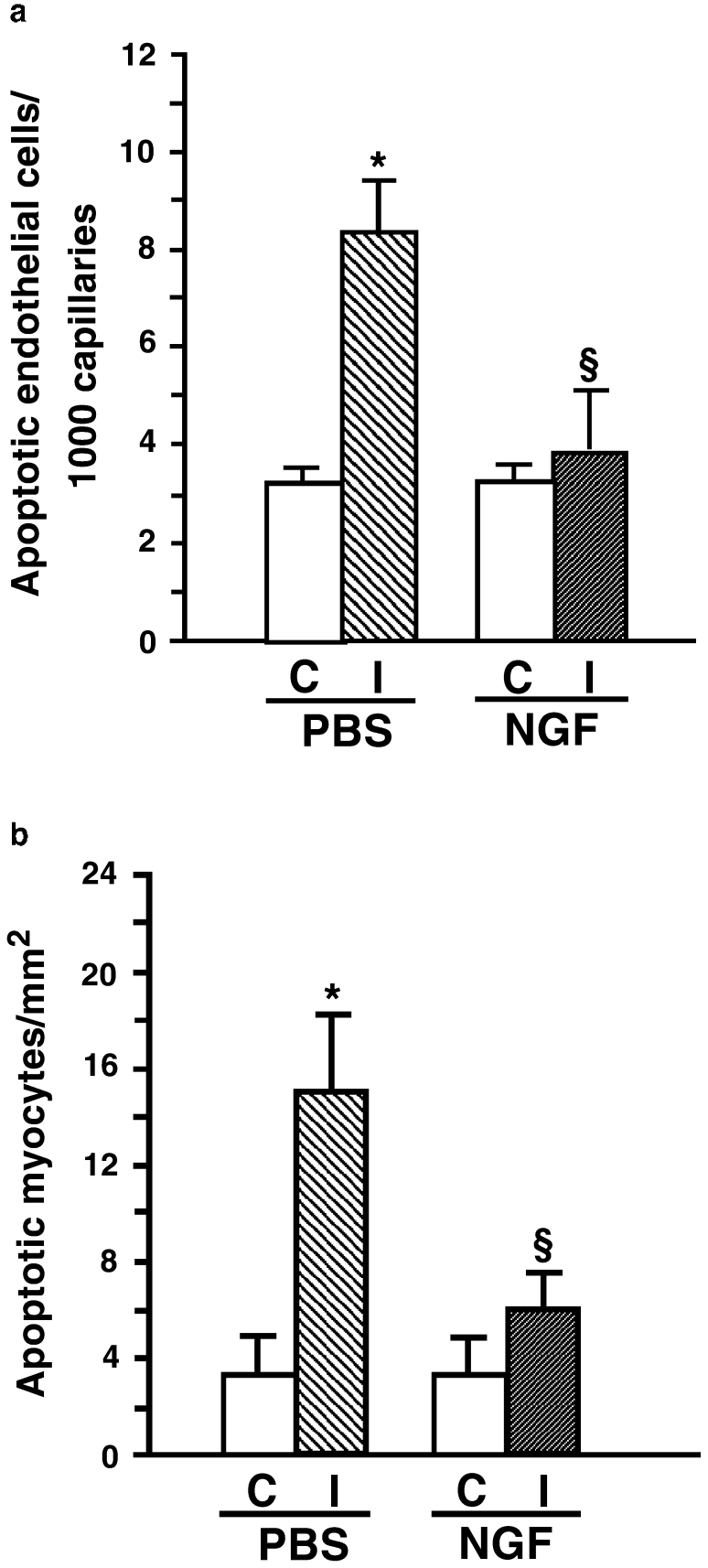

Fig. 5. The effect, in diabetic mice, of nerve growth factor (NGF) or PBS on apoptosis at the level of endothelial cells (a) or myocytes (b) in ischaemic muscles (I). Values in normally perfused contralateral adductors $(\mathrm{C})$ are shown for reference. Apoptosis was expressed as the number of TUNEL-positive endothelial cells per 1000 capillaries and as the number of TUNEL-positive myocytes per $\mathrm{mm}^{2}$ of transverse muscular section. Values are means \pm SEM. $* p<0.05$ vs contralateral; $\S_{p}<0.05$ vs PBS-injected ischaemic muscles. TUNEL, terminal deoxynucleotidyl transferase-mediated dUTP nick-end labelling

Local administration of nerve growth factor reduces p75 receptor expression. We examined whether local supplementation with NGF alters the expression of NGF receptors in ischaemic muscles of Type 1 diabetic mice. We found that the number of p75-positive endo- 


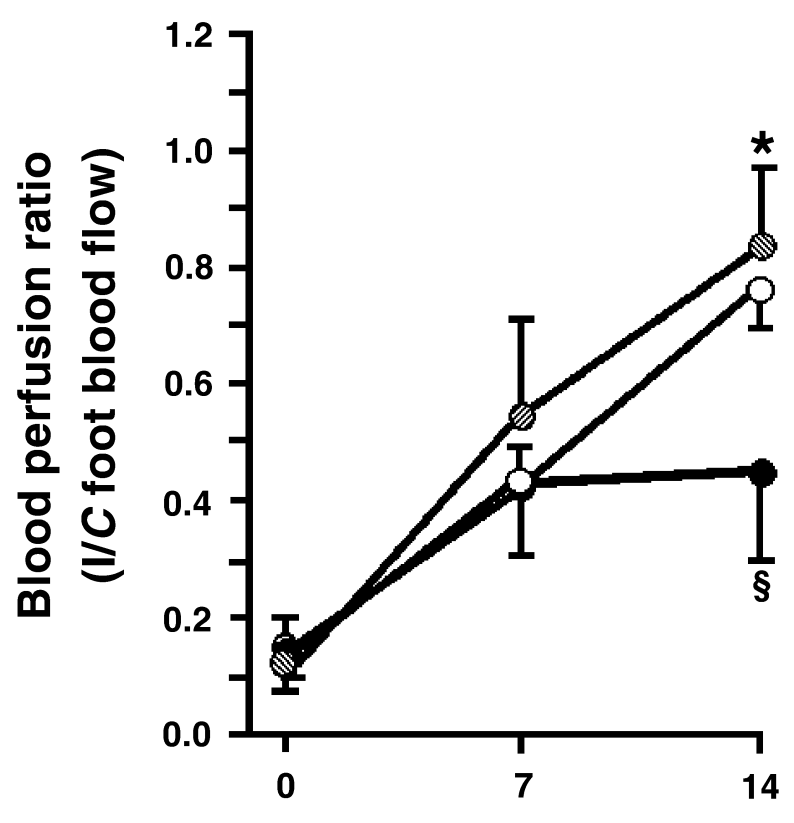

Days from ischaemia

Fig. 6. The beneficial effect of nerve growth factor (NGF) injection on post-ischaemic blood flow recovery of the ischaemic foot (expressed as left : right foot blood flow ratio $[\mathrm{I} / \mathrm{C}])$. NGF (hatched circles) or the vehicle PBS (closed circles) was injected into the ischaemic muscle of diabetic mice every day for 14 days from the moment of ischaemia induction. Non-diabetic mice (open circles) receiving PBS were used as reference. Values are means \pm SEM. ${ }^{\S} p<0.05$ vs nondiabetic mice; ${ }^{*} p<0.05$ vs PBS-treated diabetic mice
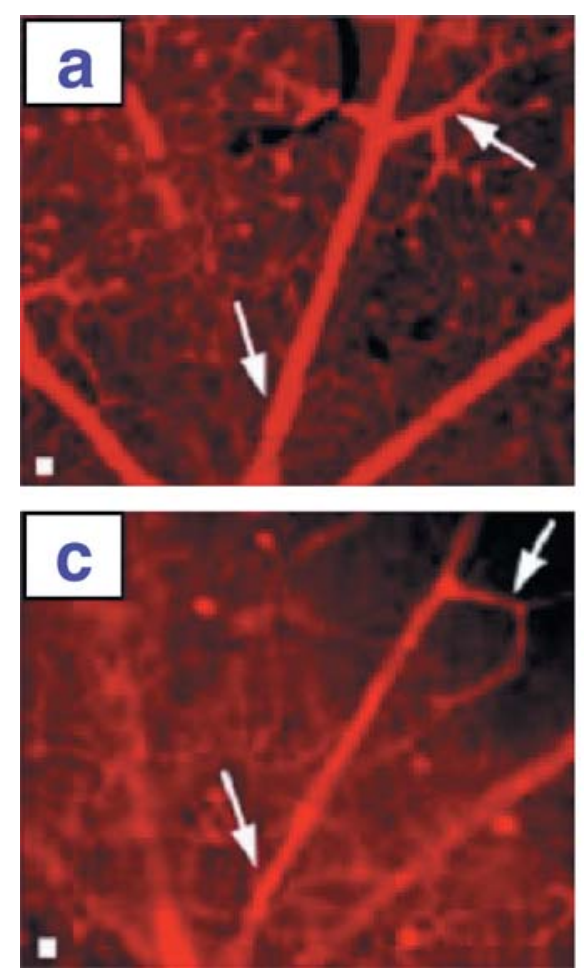

Fig. 7. The retinal vessels (arrows) in diabetic mice injected in the ischaemic adductors for 14 days with $\operatorname{PBS}(\mathbf{a}, \mathbf{b})$ or nerve growth factor (NGF) (c, d). The vessels are comparable in structure and organisation. Large vessels extend radially from thelial cells was reduced after NGF treatment $(25.0 \pm 1.9$ vs $43.4 \pm 12.4$ positive cells per 1000 capillaries in the PBS-treated group, $p<0.05)$. In contrast, the number of p75-positive myofibres was not altered by NGF $\left(5.5 \pm 3.3\right.$ vs $4.5 \pm 0.9$ myocytes per $\mathrm{mm}^{2}$ in the PBS group, $p=\mathrm{NS})$. TrkA receptor expression was not influenced by NGF administration either at the endothelial cell or the myofibre level (data not shown).

Nerve growth factor reduces HUVEC apoptosis via modulation of VEGF-A production. Under the experimental conditions used for this experiment (i.e. $24 \mathrm{~h}$ of serum deprivation and high glucose concentration), NGF did not change the total cell number $(26 \pm 4$ vs $25 \pm 3$ cells per field in PBS wells, $p=\mathrm{NS}$ ). However, as shown by Figure $8 \mathrm{a}$, NGF did promote VEGF-A production and/or release from HUVECs, as demonstrated by the increase of VEGF-A content in the medium $(1.8 \pm 0.3$ vs $0.8 \pm 0.3 \mathrm{pg} / \mathrm{ml}$ in PBS wells, $p<0.05)$. NGF reduced the rate of apoptotic death of HUVECs, this effect being nullified by a neutralising antibody for VEGF-A (Fig. 8b). Control IgG had no effect (data not shown).

\section{Discussion}

Our study shows that the expression of NGF and its p75 receptor in adductor skeletal muscles is influ-
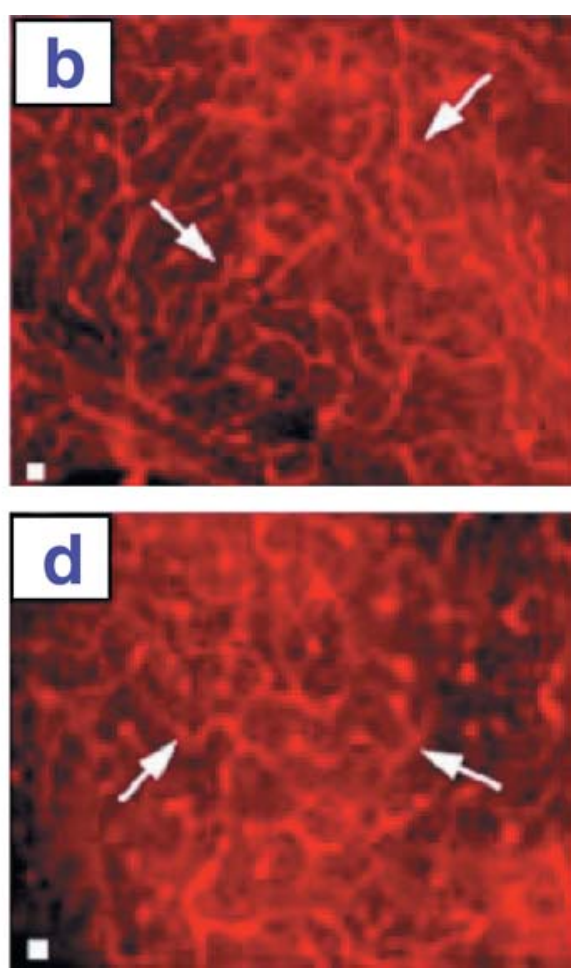

the optic disk into the central retina $(\mathbf{a}, \mathbf{c})$ and branch to form extensive microvascular plexuses within the peripheral retina $(\mathbf{b}, \mathbf{d})$. Scale box $=20 \mu \mathrm{m}^{2}$ 
a

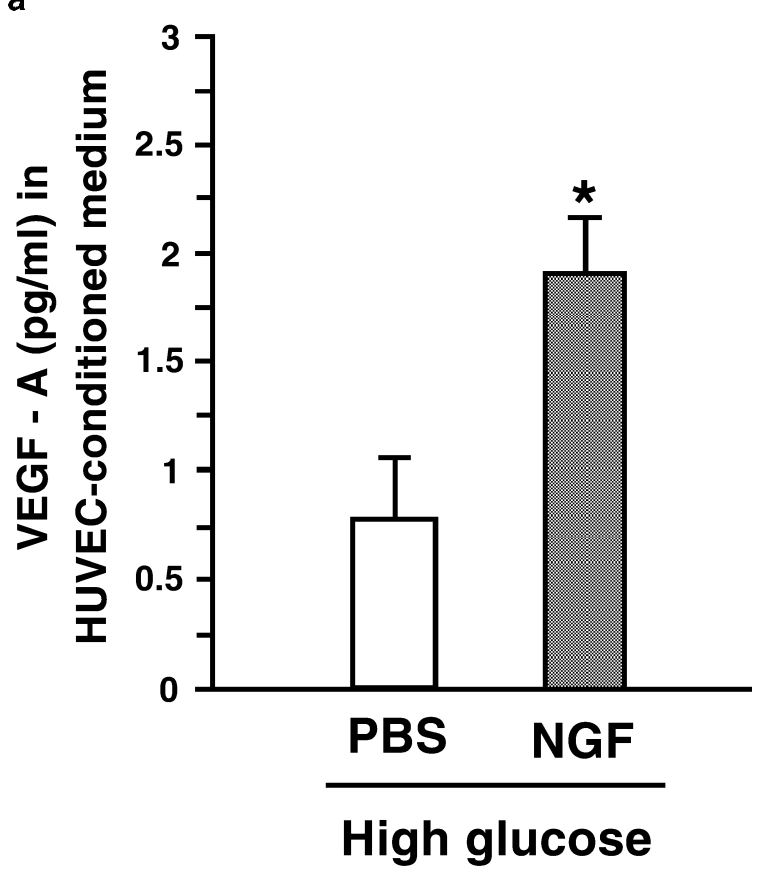

b

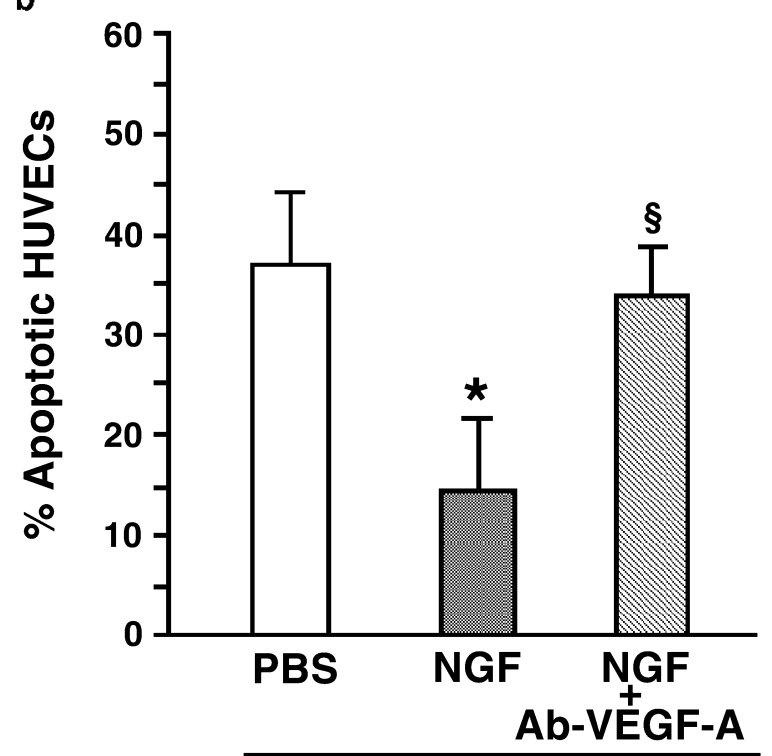

High glucose

Fig. 8. The content of human vascular endothelial growth factor-A (VEGF-A) in the conditioned medium (a) of HUVECs maintained for 24 hours in $30 \mathrm{mmol} / \mathrm{l} \mathrm{D}$-glucose (high glucose), $0.1 \%$ FBS, and either nerve growth factor (NGF) (100 ng/ml, grey column) or PBS (open column). VEGF-A was measured by a dedicated ELISA kit. Values are means \pm SEM. $* p<0.05$ vs PBS. The effect (b) of NGF (100 ng/ml, grey column) or PBS (open column) on HUVEC apoptosis caused by incubation for $24 \mathrm{~h}$ in $30 \mathrm{mmol} / \mathrm{l} \mathrm{D}$-glucose and $0.1 \% \mathrm{FBS}$. The effect of a neutralising antibody for VEGF-A on NGF action is also shown (NGF+Ab-VEGF-A, hatched column). Apoptosis was revealed by DAPI staining and expressed as percentage of apoptotic cells per total cell number per field. Values are means \pm SEM. ${ }^{*} p<0.05$ vs PBS; ${ }^{\S} p<0.05$ vs NGF alone. DAPI, 4'-6'-diaminidino phenylindole enced by Type 1 diabetes and that these changes are associated with impaired neovascularisation response to ischaemia and increased apoptosis. On the other hand, treatment with NGF tends to normalise the expressional pattern of $\mathrm{p} 75$, re-establishes reparative angiogenesis and arteriogenesis, and reduces apoptosis.

Extensive previous works have demonstrated the production of NGF by neural tissues and unravelled the effects of NGF on the central and peripheral nervous system [13]. Emerging data suggest that NGF exerts pleiotropic effects in non-neural tissues. NGF production from vascular endothelial cells has also been demonstrated $[5,14]$. In addition, NGF receptors are expressed on endothelial cells [4, 5, 14], suggesting an autocrine/paracrine loop for the neurotrophininfluencing vascular physiology. NGF may also be directly implicated in the maintenance of proper skeletal muscle phenotype, as suggested by the severe muscular dystrophy produced by NGF phenotypic knockout in adult transgenic mice [15].

Recently, we have shown that NGF acts as a potent stimulator of angiogenesis and exerts therapeutic effects on experimentally induced peripheral ischaemia in otherwise healthy mice [4]. The pro-angiogenic effect of NGF in vivo seems to be mediated by VEGF-A, as a neutralising antibody for VEGF-A inhibits the NGF-induced capillarisation response of ischaemic limb muscles [4]. Interestingly, Calza and colleagues have reported that NGF concomitantly increases VEGF-A expression and vascularity in the superior cervical ganglia of newborn rats [16]. The present study adds the new finding that under high-glucose conditions NGF stimulates human endothelial cells to produce/release VEGF-A. Moreover, we also document that the blockade of VEGF-A prevents the anti-apoptotic effect of NGF. Thus, NGF seemingly promotes cell survival via VEGF-A.

The importance of the present findings is that they indicate mutual interrelations between growth factors that control neurogenesis and angiogenesis. In vitro results are complemented by the information that NGF levels are decreased in ischaemic or contralateral adductors of Type 1 diabetic mice. NGF down-regulation might play a part in dampening the surge of VEGF-A in diabetic muscles [3] or sciatic nerves [17] subjected to ischaemia. Altogether, the above deficiencies could account for the more complicated clinical outcome observed after arterial occlusion in Type 1 diabetic mice.

NGF exerts its biological action through the contribution of two receptors: the high-affinity TrkA and the low-affinity p75 [18]. In endothelial cells, NGF-mediated TrkA phosphorylation activates the mitogen-activated protein kinase pathway and leads to cell proliferation [5]. The role and signalling pathway of p75 are still controversial. The receptor has been linked to apoptosis of vascular smooth muscle cells [19], neurons 
and oligodendrocytes [20], although consensus on this theory is not universal [21]. Our study is the first to propose a correlation between $\mathrm{p} 75$ and apoptotic death of endothelial cells, and to indicate a possible implication of p75 in diabetic microangiopathy. Up-regulation of the receptor may be a hallmark of death for diabetic tissues subjected to ischaemic insult (as shown here) or to mechanical cutaneous injury (see article by Graiani et al. in this issue of Diabetologia). The large up-regulation of p75 in endothelial cells, which occurred concomitantly with reduced NGF in the ischaemic limb muscles of Type 1 diabetic mice, may lead to an excess of p75 not occupied by their ligand. A similar scenario has been implicated by some authors in the generation of apoptotic signals in neurons $[22,23,24,25]$. Moreover, the interaction between NGF and TrkA, as well as post-receptorial TrkA signalling, may be affected by reduced NGF and increased p75 expression. Additional work will be necessary to clarify whether p75 has a direct proapoptotic effect on endothelial cells and to identify the molecular signalling that leads to activation of p75 in diabetic endothelial cells.

Most importantly, in a diabetic mouse model with superimposed ischaemia, we demonstrated that NGF supplementation stimulates reparative neovascularisation, improves blood flow recovery and concomitantly decreases the number of p75-positive endothelial cells and apoptosis. Interestingly, with regard to possible clinical exploitation, repeated local administration of NGF into ischaemic limbs did not endanger the retina of mice. This is not a secondary issue, as it is well known that abnormal capillarisation causes retinopathy, one of the most diffuse and severe complications of diabetes. Moreover, we did not observe any evident signs of abnormal behaviour connected to increased pain perception in NGF-treated mice.

In conclusion, our study suggests that NGF may represent a potential new therapeutic tool to combat microvascular complications caused by diabetes.

Acknowledgements. This study was supported by grants from the Juvenile Diabetes Research Foundation (JDFR, USA No. 1-2001-877) and from the Italian Ministry of Scientific Research and University (MIUR-FIRB project New Biotechnological and Post-Genomic Tool to Combat Ischaemic Disease). The authors thank Dr L. Aloe (Consiglio Nazionale delle Ricerche, Rome) for providing purified murine NGF.

\section{References}

1. Kannel WB, McGee DL (1979) Diabetes and cardiovascular disease. The Framingham study. JAMA 241:20352038

2. Currie CJ, Morgan CL, Peters JR (1998) The epidemiology and cost of inpatient care for peripheral vascular disease, infection, neuropathy, and ulceration in diabetes. Diabetes Care 21:42-48
3. Rivard A, Silver M, Chen D et al. (1999) Rescue of diabetes related impairment of angiogenesis by intramuscular gene therapy with adeno-VEGF. Am J Pathol 154:355364

4. Emanueli C, Salis MB, Pinna A, Graiani G, Manni L, Madeddu P (2002) Nerve growth factor promotes angiogenesis and arteriogenesis in ischemic hindlimbs. Circulation 106:2257-2262

5. Cantarella G, Lempereur L, Presta M et al. (2002) Nerve growth factor-endothelial cell interaction leads to angiogenesis in vitro and in vivo. FASEB J 16:13071309

6. Hellweg R, Hartung HD (1990) Endogenous levels of nerve growth factor (NGF) are altered in experimental diabetes mellitus: a possible role for NGF in the pathogenesis of diabetic neuropathy. J Neurosci Res 26:258267

7. Anand P, Terenghi G, Warner G, Kopelman P, WilliamsChestnut RE, Sinicropi DV (1996) The role of endogenous nerve growth factor in human diabetic neuropathy. Nat Med 2:703-707

8. Apfel SC (2002) Nerve growth factor for the treatment of diabetic neuropathy: what went wrong, what went right, and what does the future hold? Int Rev Neurobiol 50:393-413

9. Fernyhough P, Diemel LT, Hardy J, Brewster WJ, Mohiuddin L, Tomlinson DR (1995) Human recombinant nerve growth factor replaces deficient neurotrophic support in the diabetic rat. Eur J Neurosci 7:1107-1110

10. Emanueli C, Salis MB, Pinna A et al. (2002) Prevention of diabetes-induced microangiopathy by human tissue kallikrein gene transfer. Circulation 106:993-999

11. Jiang B, Behzadian MA, Caldwell RB (1995) Astrocytes modulate retinal vasculogenesis: Effects on endothelial cell differentiation. Glia 15:1-10

12. Brooks SE, Gu X, Samuel S et al. (2001) Reduced severity of oxygen-induced retinopathy in eNOS-deficient mice. Invest Ophthalmol Vis Sci 42:222-228

13. Levi-Montalcini R, Skaper SD, Dal Toso R, Petrelli L, Leon A (1996) Nerve growth factor: from neurotrophin to neurokine. Trends Neurosci 19:514-520

14. Tanaka A, Wakita U, Kambe N, Iwasaki T, Matsuda H (2004) An autocrine function of nerve growth factor for cell cycle regulation of vascular endothelial cells. Biochem Biophys Res Commun 313:1009-1014

15. Ruberti F, Capsoni S, Comparini A et al. (2000) Phenotypic knockout of nerve growth factor in adult transgenic mice reveals severe deficits in basal forebrain cholinergic neurons, cell death in the spleen, and skeletal muscle dystrophy. J Neurosci 20:2589-2601

16. Calza L, Giardino L, Giuliani A, Aloe L, Levi-Montalcini R (2001) Nerve growth factor control of neuronal expression of angiogenetic and vasoactive factors. Proc Natl Acad Sci USA 98:4160-4165

17. Schratzberger P, Walther DH, Ritting K et al. (2001) Reversal of experimental diabetic neuropathy by VEGF gene transfer. J Clin Invest 107:1083-1092

18. Yano H, Chao MV (2000) Neurotrophin receptor structure and interactions. Pharm Acta Helv 74:253-260

19. Wang S, Bray P, McCaffrey T, March K, Hempstead BL, Kraemer R (2000) p75(NTR) mediates neurotrophininduced apoptosis of vascular smooth muscle cells. Am J Pathol 157:1247-1258

20. Majdan M, Walsh GS, Aloyz R, Miller FD (2001) TrkA mediates developmental sympathetic neuron survival in vivo by silencing an ongoing p75NTR-mediated death signal. J Cell Biol 155:1275-1285 
21. Nichols A, Martinou I, Maundrell K, Martinou JC (1998) The p75 neurotrophin receptor: effects on neuron survival in vitro and interaction with death domain-containing adaptor proteins. Apoptosis 3:289-294

22. Aloyz RS, Bamji SX, Pozniak CD et al. (1998) p53 is essential for developmental neuron death as regulated by the TrkA and p75 neurotrophin receptors. J Cell Biol 143:1691-1703

23. Bronfman FC, Tcherpakov M, Jovin TM, Fainzilber M (2003) Ligand-induced internalization of the p75 neu- rotrophin receptor: a slow route to the signaling endosome. J Neurosci 23:3209-3220

24. Chao M, Casaccia-Bonnefil P, Carter B, Chittka A, Kong H, Yoon SO (1998) Neurotrophin receptors: mediators of life and death: Brain Res Rev 26:295-301

25. Lopez-Sanchez N, Frade JM (2002) Control of the cell cycle by neurothrophins: lessons from the p75 neurotrophin receptor. Histol Histopathol 17:1227-1237 